\title{
Piano Grading Test in Eastern China-A Case Study on Nantong
}

\author{
Guo Yun \\ Nantong University, Jiangsu, China
}

\begin{abstract}
The universal education and basic education of piano in China have entered an era of rapid development. Parents are willing to invest in their children's art education if only they can afford it, which has become a trend throughout the nation. Nantong is a city in Jiangsu Province located in eastern China. As a member of Yangtze River economic belt, its uplifting economy and massive urbanization have improved the cultural taste of the whole city. Therefore, piano education has already blended into the spiritual life of citizens. Countless training institutions have been established to meet the market demand. Meanwhile, all sorts of art grading tests turned up, contending for students. This paper focuses on the piano grading tests in this area, digs into their specific features, and tries to analyze their status quo and future development. From that single point, a wider horizon of art grading tests can be speculated, which hopefully will attract the attention of other researchers.
\end{abstract}

Keywords: regional, piano, grading, status quo, future development

\section{Introduction}

Piano education can improve learners' character and perseverance. With the increasing number of learners and piano amateur, playing testing and evaluating system is becoming more complete and developed. By researching and reflecting on the status quo of piano grading tests, this paper aims to have a true understanding of this booming situation, so as to offer some theoretical basis and practical advice for the graded teaching. In those areas where economy development level is high, the relatively profound education resources and the huge need together lead to a giant industrial chain around piano. All kinds of training institutions and art grading tests spring up like mushrooms. Because of the uniqueness and potential advantage of basic piano education, lots of professionals and non-professionals devote themselves in that career, contending for students. That phenomenon means both opportunity and challenge for the parents and students. Apparently, what the market needs most is knowledge and regulation.

\section{A Brief Review on Music Grading Tests Abroad}

The domestic music grading system originates from abroad. The recorded history of music grading system can be traced back to 100 years ago. The following three countries will be taken as examples: Great Britain, Japan, and Canada. Analysis and comparison will be executed to explain the origination and history of music grading.

The music grading system is invented by the British. The External Exam Board is established in 1889, merged by two famous academies: Royal College of Music and Royal Academy of Music. The board is aimed at maintaining an equal, justified, and regulated environment for the judgment on the periodical progress of the

Guo Yun, M.A., associate professor, College of Fine Arts, Nantong University. 
students. Nowadays, the core of the board has been developed into the unification of the grading standard. It standardizes the grading procedures and encourages the quality-oriented education of music.

An annual piano grading test in Japan is organized by National Piano Tutor Association of Japan. The candidates can sign up in different districts for multiple levels. The piano grading test has different forms: solo, duet, and double piano. The candidates can write and perform melodies by themselves. The judges will grade according to the difficulty, impression, and complexity of the production. Due to the flexible and liberal guideline, the passing rate of piano grading in Japan is relatively high and outstanding candidates will be honored and awarded.

As for Canada, there is only one music grading institution, namely, Royal Conservatory of Music (RMC). It has a history of more than 100 years. Grading tests will be held in winter, spring, and summer, once a season. Levels include "Beginner level,” "Level 1 to Level 10," "Performer level,” and "Tutor level.” The certification of "Tutor level" means qualification for teaching corresponding courses. Therefore, RCM is a test closely related to qualifications for music education. There is a wide range of optional melodies. Diversity, enjoyment, and era sensation are emphasized. In the comprehensive quality section, other than Solfeggio and visual play, the candidates' comprehension of basic musical theories will also be examined, which reflects the emphasis on the comprehensive quality of candidates.

\section{The Status Quo of Piano Grading Tests in Eastern China}

\section{The Grading Institution in Eastern China}

On the basis of stable political environment and rapid economic development, the pursuit of art becomes a necessity of everyday life. A lot of prominent Chinese pianists, such as Langlang, Li Yundi, Wang Yujia, and Chen Sa, have displayed their glamour on the international stage, which contributes to the prevalence of piano education and the favor among the public. Learning piano has become a fashion and a phenomenon of the times. Along with the normalization of piano education, piano grading tests become a key link of piano education in China. Now, as everyone has the opportunity to learn piano, there comes an urgent need of a grading system to judge performing levels. As a result, the professional conservatories all over the country became the first "judges" of piano grading tests. The piano grading system has close connections with economic, politic and many other domains. Because of the vast acreage of China, these conservatories lie in different areas. Therefore, their banks of formulation music and the grading systems created by different committees also differ from each other.

The grading institutions in eastern China can be divided into two types: trans-provincial musical grading institutions and provincial grading institutions. The trans-provincial musical grading institutions include some famous conservatories and national-level specialized associations, such as Central Conservatory of Music, China Conservatory of Music, Shanghai Conservatory of Music, China National Opera \& Dance Drama Theater, and Chinese Musicians' Association. They have exam sites in many major and medium cities across the nation. The provincial musical grading institutions cover all the cities in a province. These institutions are mainly provincial conservatoires and specialized associations, such as Nanjing University of the Arts, Musicians' Association of Jiangsu Province, Zhejiang Conservatory of Music, and Musicians’ Association of Zhejiang Province. 


\section{Normalization of Piano Grading Tests: The Social Impact}

The establishment of piano grading test plays an important role in the socialization and popularization of piano education. It has a positive impact on the advancement of social artistic level. The appearance of the grading system had met the market demand of piano education, and promoted a relatively regulated environment for musical aesthetics. One major target of piano grading test is to examine the periodical learning condition of the students. Another is to checkout the teaching results of the piano tutors. Students will practice a lot of formulation music with tutors' instructions, by which the quality of tuition can be improved, the bank of formulation music can be enriched, and the students can learn from a more systematic perspective with interests. Meanwhile, the grading system can also select potential outstanding musicians. The piano education requires the development of piano grading system. In return, the piano grading system then normalizes and improves the level of aesthetic taste for music among the public, leading to an enormous demand of artistic talents in the society.

\section{The Defects Caused by Excessive Expansion of Piano Training Industry}

As the piano grading tests had been rapidly popularized all over the nation, people's recognition of the piano grading system somehow became tilted. Influenced by the exam-oriented basic education in China, the assessment of artistic quality tends to be extremely utilitarian. Parents are willing to flow with the tide and have no definite views. Ignoring the uniqueness and age of their children, most of them urge the tutors to help their children pass the grading tests, from "Initiator level” to "Level 10," within the primary school period. As far as they are concerned, the aim of learning to play the piano is only to get a Level 10 certification. Parents often promise their children that once they passed Level 10, they will not have to learn to play the piano anymore. In addition, many parents are attracted by bonus points in entrance examinations or the potential development of intelligence. What is more, the only reason why some children learn to play the piano is to realize their parents' musical dream that can never come true for them. Even though these goals probably can be achieved through piano education, learning to play the piano with these preexisting purposes can only lead to the ignorance of the essential meaning of music education - the love for music. It is the enjoyment of sweet melodies that come first. The day that the parents show off the certifications of their children sees the distortion of the piano grading tests and the corruption of music education.

Where there is demand, there is a market. The piano training industry had permeated into every corner of cities. Training organizations are literally everywhere and the number is keeping increasing. Some classrooms cover an area of 300 to 800 square meters, with glorious and luxurious decorations. Others may only have 20 to 50 square meters, located in an underground garage while declaring itself as "training center." Moreover, the qualities of the teachers vary. Besides the specialized teachers from standard conservatoires and institutions, many graduates and undergraduates, even some totally outsiders were attracted by the immense potential profits, striving to invest in piano education and establish schools. As for those musical instrument stores which offer training courses with sale missions, they will provide tutors if only you buy their productions. Teachers who are capable of playing multiple instruments are popular in the market. On the other hand, their advertisements are pervasive. They can expertly use we-medias, such as WeChat and Websites, as their tools, operating endless commercial marketing. These training organizations are contending for the qualifications as second-hand or third-hand agency of grading institutions. To achieve that goal, they have been roping teachers and expanding their business scope. Standards have been twisted to meet the needs of all kind of learners with 
different motivations. Education is the root of ethics. Thus, the training organizations and artistic grading institutions ought to emphasize on and consciously lead the normalized and positive development of piano education.

\section{An Analysis on Piano Grading Tests in Nantong}

Nantong is a city lying in the northeast of Jiangsu Province, in the north of Yangtze River delta. It is one of the earliest 14 coastal cities that open to the outside world. It stretches to the Yellow Sea in the east, and borders on Yangtze River in the south. With Taizhou in the west and Yancheng in the north, it is adjacent to Shanghai and Suzhou. Nantong is an uprising young city. Recently, it meets a rapid economic development because of its unique geographic location and its status as a major transportation junction. As a result, the residents of Nantong have been increasingly focusing on their life quality and aesthetic needs, which indirectly promoted the development of non-official training organizations and music education industry. Along with the development of music education, related industries entered a rapid developing spring. The sales of the musical instrument stores in Nantong had an obvious leap from tepid to gratifying. Piano became one of the family necessities with a powerful momentum.

According to a survey, the recent sale volume of pianos in Nantong is around 3,000 annually. The producers include all range of levels, from common to excellence: Kawai and Yamaha from Japan, Samick and Seiler from Korea, Steinway from America, Germany producers, home-bred brands Zhujiang and Langlang, and so on. The sale volumes of all these producers are increasing every year and the trend continues. Lately, the Ministry of Education issued a notification named Some Suggestions to Advance the Development of Artistic Education in Schools, which the ministry declared in 2015, the appraisal of artistic quality will be included in the references of high school and college entrance examinations. It directly announced the bonus point's policy in high school entrance examination that the students who passed Level 9 tests of artistic grading will get three or more points in the entrance examination. In reaction, many artistic training organizations began to put forward a lot of artistic grading projects, expanding their scale of teaching. Most of the parents are willing to foster the artistic quality of their children and enhance their intelligence and emotion quotients from childhood. Therefore, they tend to choose piano. Some other parents just want to seek quick success and ask their children to learn to play the piano compulsorily with the hope to get the bonus points or take piano as a career in the future. All these intentions leaded to the long-lasting "piano-craze" among children and teenagers in Nantong. Meanwhile, the "grading test craze" in Nantong is also spreading with a horrible speed.

\section{The Provincial Grading Institutions That Sited in Nantong}

Musicians’ Association of Jiangsu Province has been holding grading tests since 1991. It organizes a performance by outstanding candidates every year. What is more, it also regularly provides various normal courses, like Piano Tutors' Seminar. According to the survey data, there were more than 1,000 candidates who learned to play the piano, saxophone, Chinese zither, Er-hu, and other 20 kinds of instruments in the grading tests held by Musicians' Association of Jiangsu Province. A lot of these candidates applied for a piano certification. The exam sites were set in high schools, middle schools, and primary schools.

The social artistic level grading test held by Nanjing University of the Arts is established in 1996. It is one of the earliest social artistic level grading tests approved by the Ministry of Culture. It is a comprehensive grading test, containing different fields of arts like music, painting, calligraphy, dance, and recitation. Music 
grading, for instance, has four sub-categories: western instruments, Chinese instruments, percussion, and vocal music. The music grading tests are only held once a year in the middle of August, during the summer holiday. Survey data reveals that 2,000 to 3,000 candidates who learn the piano took the grading test held by Nanjing University of the Arts every year, let alone other candidates. Except for piano, the number of candidates for certification of guitar, Chinese zither, drum set, and some other instruments are also growing annually.

\section{The Trans-Provincial Institutions That Sited in Nantong}

Social artistic level grading tests held by trans-provincial institutions, such as Central Conservatory of Music, China Conservatory of Music, Shanghai Conservatory of Music, and China National Opera \& Dance Drama Theater, also set exam sites in Nantong. They organize grading tests once or twice a year. The subject of tests concentrate on Chinese and foreign instruments. The number of candidates for piano certification is around 2,000, which includes repetitive candidates (candidates who apply for artistic levels in two to three different exam sites).

\section{Features of Piano Grading Tests in Nantong}

As for grading approach, all these grading institutions listed above have something in common. That is, the music used for grading must be chosen from a fixed bank of melodies assigned by the grading institutions. The banks may differ, but their levels of difficulty are close to each other.

First and foremost, musical scale and arpeggio are basic skills that must be examined. Secondly, the institutions often choose etudes for tests from melodies written by Carl Czerny, Moszkowski, Chopin, and Debussy. Polyphonic music usually includes those written by Bach, Minuet, Inventions et Sinfonias, English Suites, French Suites, and The Well-Tempered Clavier. Last but not least, as for those medium and major works, candidates may choose from a wide range of melodies. The bank covers the masterpieces of different ages and all kind of styles, such as Rondo written by Beethoven, Moments Musicaux written by Schubert, and In Murcia's Garden written by Joaquin Turina Perez. During the grading, the judges may interrupt candidates' performance according to the situation without affecting the result of the grading. From Level 8 to Level 10, theoretical exams will be added into the tests. After the grading tests, the unified level certifications made by responsible institutions will be issued two to three months later. The grading standards include four grades: excellent, favorable, qualified, and unqualified.

When the music grading system was introduced to Nantong for the first time, candidates were mainly teenage students under 18 years old. The age and identity of the candidates has considerably changed through the developments of these years. Although teenage students are still the mainstream of the candidates, people from other walks of life also begin to take part in the tests, including housewives, white-collars, and even retired elders. According to the survey data, of all the social artistic grading tests, music-grading tests take a large scale. The piano grading tests, as a matter of fact, is the main force of music grading tests. In Nantong, which is honored as "the first city in modern China," the number of the candidates participating in the grading tests is large, which reflects people's preference to the investment in non-degree-related basic education like artistic education and piano education. Indeed, it is widely recognized that learning to play the piano requires multi-tasking: eyes, hands, and brain must cooperate coordinately, where the learners can cultivate their tastes while develop their brain by improving their retention and manual dexterity.

The grading tests not only examine the periodical results of a learning process, but also facilitate the consistent development of relative industry chains. 


\section{Piano Grading Tests in Nantong: Challenges and Their Origination}

Challenges that piano grading tests in china are facing with. A paper written by Lu (2012) entitled "A survey on amateur piano education in Hohhot," where the author describes some long-existing problems of piano grading tests in Hohhot. The following remarks are quotations of his paper:

The parents see the grading tests as a tool to show off the ability of their children (Lu, 2012). They like to compare their children with others. That kind of parents takes grading tests as the ultimate aim of learning to play the piano. In order to make sure that their children will pass the examination, the parents will be excessively strict on the training of piano. Once the children made a mistake, accusations, or even physical punishments will come to them. Thus, the proper pride of the children will be destroyed. Their interest in learning to play the piano will be cut down. Besides, teachers also have a kind of negative attitude toward grading tests. Some teachers insist that every learner must take the test, just to ensure their consistent students stream.

Mou (2008) explained the main challenges that piano grading tests in Shandong Province met in her paper entitled "A survey on piano grading tests in Shandong Province: Actuality and reform ideas." She pointed out that:

1. The management of the whole organization is not regulated. The grading tests in some cities lowered the grading standard, just to gain more profit. Thus, the grading procedures cannot be strictly carried out. Moreover, the grading standards made by the cities and the province differ. Some judges are likely to give more points to those who are familiar with them. Besides, the certifications are issued by different institutions, which brought about the decrease of the authority of the grading tests.

2. Tutors are not aware of their teaching aims. Many students, parents, and teachers have a vague cognition upon the aims and meaning of grading tests. Some teachers blindly pursue progression.

3. Tutors are not capable of helping their students analyze and comprehend the melodies. Teaching and learning are the two significant ends of grading tests. The good teachers and poor teachers are intermingled in the piano education industry. A lot of problems had been exposed during the tuition.

4. The grading standards are uncertain. All kinds of people engage in the teaching profession and their abilities differ. The lack of a unified standard leaded to the huge gaps between certificated students in different cities.

In a paper entitled “A simplified analysis on the amateur piano grading tests in Jiangxi Province,” Zeng and Xie (2013) interpreted the problems of amateur piano grading tests in Jiangxi Province:

1. The organization of some piano grading institutions is not regulated. There are five or six grading institutions in Jiangxi province. Each has their own grading system and standards.

2. The most urgent problem is the utilitarianism of the students and parents. As more and more parents begin to pay attention to the early music education of their children, some alarming phenomenon appeared. In the high school entrance exams of some cities, grading tests are bound with bonus points for students (Mou, 2008) specializing in art. Some learners dream of overnight fame by means of talent shows (Zeng \& Xie, 2013).

3. The qualities of the tutors vary. Some training organizations had no qualified teachers. Motivated by profits, they hired many unprofessional music teachers to guide the students, which is a severe disturbance to the environment of amateur piano education.

4. The updating of music library lags behind. The choice of melodies lacks multiplicity. In most cities of Jiangxi Province, the grading textbooks used by amateur piano grading tests are the one that edited by Chinese Musicians' Association. The textbook is fixed, thus, the choice of melodies lacks multiplicity. The piano grading tests in Jiangxi province started in 1993. Only four editions of textbooks were used in that 20 years. The updating of the textbooks is too slow and there is lack of multiplicity in melodies. In contrast, grading institutions in Guangdong province and Shanghai edit their textbooks every year, in March or April, to prevent the students from blindly practicing the grading melodies and ignoring the basic training.

From the three papers above, we can find that different problems exist in the piano grading tests of different districts. So, what about the piano grading tests in Nantong? 
Problems of grading tests in Nantong. The most common problem of grading tests in Nantong is the sightless grade-skipping. One should follow the scientific procedures no matter what knowledge he/she is learning. As for grading tests, students should choose proper levels according to their ability. Under normal circumstances, one can decide which level of test is proper only when he/she feels comfortable playing those melodies appointed as formulation music of that level. If the students blindly skip the grades, they can never get sufficient development. Some training organizations will begin to tutor the children since their second year in kindergarten according to their parents' requirement. Some parents want their children to pass Level 6 in Grade 1 and Levels 8 to 10 in Grades 3 and 4. That kind of utilitarianism results in a lack of comprehensive knowledge of piano. Most of these students will meet with many problems as follow:

1. Their fingers could not stand steady;

2. They are not familiar with basic skills;

3. They seldom pay attention to expression marks;

4. They often play a false note;

5. they have no comprehension and feelings of their own.

The chaos of piano grading institutions, grading textbooks, and grading standards in Nantong are the problems to be solved. In fact, in order to hold some social artistic activities, to improve the comprehensive cultural skills of the students, and to promote their reputation and enrollment rate, many schools in Nantong recruited a lot of students with a specialty, which is proven with a high-level certification. Moreover, the artistic bonus point's policy (students who passed artistic Level 9 tests will get three more points in high school and college entrance examinations) has been issued throughout the country this year. These policies have attracted some students to join in the piano grading group. Meanwhile, some parents asked the tutors to quicken the teaching progress, just to ensure that their children can have a certificate in time.

There are more than 10 grading institutions in Nantong. Each assigned their own grading formulation music. Since these institutions have similar levels, it is hard for the public to judge which one is more authoritative and scientific. Take Chongchuan District of Nantong City as an example, we visited many piano training organizations and musical instrument stores there, and found that there are three to four artistic training organizations in almost every street, especially those streets near schools. These organizations and stores are agencies of different grading tests, which is one of the approaches to get more students. Some exam sites even promise that every candidate can pass the tests. They increase the passing proportion of the tests to improve their fame and popularity. During the survey, some parents said that their children finished a grading test within one minute and passed it.

The piano grading tests in Nantong are mainly held by three institutions: Shanghai Conservatory of Music, Nanjing University of the Arts and Musicians' Association of Jiangsu Province. Although different grading tests from Level 1 to Level 10 are close to each other in difficulty, the melodies still vary. The preference of the judges, the blindness of the parents and the direction of the public opinions all have great influence on the choice of the candidates. Nowadays, the piano grading textbooks in Jiangsu province still has not been unified yet. The regional judges are changing every year. These unfixed and flexible factors lead to the uncertainty of the grading value. Therefore, the students and their parents are easy to feel confused and hesitated. Apparently, grading results concluded by such approach could not be used as the "basic line” to measure whether one's quality-oriented education meets the requirements. 
The utilitarianism of piano grading tests in Nantong is another problem to be solved. Many parents in Nantong send their children to learn to play the piano for two reasons: To exploit the intelligence of their children and to invest the future. Most parents are looking forward to the success of their son or daughter. However, being excessively anxious for success can only become an obstacle of it. Some parents wish their child to be better than others of his or her age. They are keen on comparison and the pursuit of grading levels, which lead to the disordered and unreasonable progress of the training. They hope that their children can pass Level 10 before middle school. Therefore, some parents blindly urged the tutors to quicken the teaching procedure of their children. The children were asked to play exclusively formulation music that year, keeping practicing the same formulation music everyday. Thus, the proper learning progress and arrangements are broken. Children will soon be tired of playing the piano. What is more, some parents choose tutors unreasonably by their passing rate, ignoring the individual uniqueness of every child.

The development of everything follows a route from mania to the earliest and most essential needs. So, is the learning of playing the piano. If you make the mistake once you aimed at results and levels from the beginning, your efforts will be used in wrong direction. Fortunately, in the powerful stream of grading-oriented education, there are still many tutors who insist on the morality of their profession. They arrange the grading tests or competitions according to the students' actual proficiency and progress of learning. Thus, the confidence and the performing ability of the students will be advanced. On the other hand, some children and their parents never take the grading tests though their performing ability and musical expression are undeniably professional. These children are undoubtedly in love with music. They learn to play the piano from step to step, from enlightenment to the capability for performance to some degree. Grading tests are only periodical assessments to them, but not the crux that determined their whole life. There are many learners who never touch their pianos since they passed Level 10. They have forgotten their original motivation to learn to play the piano. When they started to play the piano, they thought the music of piano would become a lifelong companion, but it did not.

\section{Suggestions on the Piano Grading System}

\section{Expedite the Scientific, Regulated, and Systematic Construction of Piano Grading}

Now, many candidates in China are willing to skip the grades. They will not follow the scientific rule to learn to play the piano, which will lead to intonation errors, unstable rhymes, and missing phrases in the grading tests. All in all, they lack consciousness of musical beauty. In order to prevent the grade-skipping phenomenon, the authority should expedite the scientific construction of the piano grading system. The piano grading system in China must be under the supervision of the government. The piano grading system should be more reasonable and regulated to establish a unified piano grading institution and to issue unified textbooks and grading standards. As we have entered the century of internet, network-based management should be brought in, which can ensure the transparency of grading information. We can also use the internet to record the process of grading tests, which can supervise the equality and fairness of it. In short, we should expedite the scientific, regulated, and systematic construction of piano grading, as soon as possible.

\section{Value the Assessment of Basic Skills and Musical Accomplishment Equally}

If the music skills of the candidates are the only concern in the tests, candidates may neglect the aesthetics of music and will hardly improve their musical accomplishment. Some parents and teachers have no clear idea about the significance of musical accomplishment. Learning to play the piano is a kind of knowledge, interest, 
and musicianship to the learners. Piano is a sort of music, while music is a discipline of art. The function of music is to stimulate people's cognition of beauty, and improve their aesthetic ability. Therefore, music education aims to arouse learner to appreciate the beauty while learning music knowledge and skills, only by which the music education can be advanced.

\section{Establish a Unified Criterion for Grading Institutions, Textbooks, and Standards}

The grading institutions in China are not unified. Thus, the grading textbooks and grading standards are varied. Certifications issued by different institutions in different districts have different applicability, which has a long-term effect on the development of piano grading in the future. Therefore, we should establish a unified criterion for grading institutions, textbooks, and standards. Different grading policies should be applied for different districts according to the different situations, which will make the piano grading system more regulated, equal, and fair.

\section{Conclusion}

In response to the existing problems of the piano grading actuality in Nantong, this paper gathered, analyzed, and summarized the survey data by means of investigation, documentary, and case-study methods. Limited by personal ability and external conditions, the survey data is somehow not comprehensive. However, it is still representative in a certain range. The problems discovered are not purely regional, but also universal and representative. We hope that a unified social art-grading criterion will soon be established, which will provide a regulated and abundant music bank and function as a responsible guider and supervisor.

\section{References}

Lu, M. Y. (2012). A survey on amateur piano education in Hohhot. Hohhot: Inner Mongolia Normal University.

Mou, L. (2008). A survey on piano grading tests in Shandong province: Actuality and reform ideas. Qilu Realm of Arts, 5, 15.

Zeng, J., \& Xie, L. (2013). A simplified analysis on the amateur piano grading tests in Jiangxi province. Shenzhou, 34, 15. 